\title{
Obesity and Sarcopenia in Survivors of Childhood Acute Lymphoblastic Leukemia
}

\author{
Payal Malhotra, ${ }^{1}$ Gauri Kapoor, ${ }^{1}$ Sandeep Jain, ${ }^{1}$ Silky Jain, ${ }^{1}$ Anurag Sharma ${ }^{\mathbf{2}}$ \\ From Departments of ${ }^{1}$ Pediatric Hematology Oncology and ${ }^{2}$ Biostatistics, Rajiv Gandhi Cancer Institute and Research Centre, \\ Rohini, Delhi, India.
}

Correspondence to: Dr Gauri Kapoor, Department of Pediatric Hematology Oncology, Rajiv Gandhi Cancer Institute and Research Centre, Rohini Sector 5, Delhi,

India.kapoor.gauri@rgcirc.org

Received: May 26, 2020;

Initial review: June 29, 2020;

Accepted: September 17, 2020.
Objective: To describe the prevalence of obesity and sarcopenia among survivors of childhood acute lymphoblastic leukemia (ALL) using DEXA scan, and study associated risk factors. Methods: This case control study was conducted between July, 2013 and June, 2014 at a tertiary care cancer centre in India. Study participants included 65 survivors of childhood ALL who were $<18$ years of age at diagnosis, treated between years 1996 and 2008 , and were at least two years since completion of therapy. The controls included 50 matched siblings. Dual energy $X$-ray absorptiometry (DEXA) was used to study the body composition (body fat percentage, $\mathrm{BF} \%$ and lean body mass, $\mathrm{LBM}$ ) of the participants and controls. McCarthy's body fat reference data were applied and logistic regression analysis was used to study various risk factors. Results: At a median (range) follow-up of 5 (7.217.2 ) years, $B F \%$ (DEXA) identified a significantly higher prevalence of obesity of $21.5 \%$ $(14 / 65)$ and sarcopenic obesity (14\%) among survivors as compared to the controls $(0 / 50$, $P<0.001)$, while the prevalence of sarcopenia as detected by LBM was similar at $60 \%$ (39/ $65)$ and $56 \%(28 / 50)$, respectively. On multivariate analysis, age at evaluation, high-risk disease and cranial irradiation were independently associated with high likelihood of obesity, while none of the factors predicted sarcopenia. Conclusion: High prevalence of obesity and sarcopenic obesity were observed among survivors of childhood ALL.

Keywords: Body composition, Body fat, DEXA, Lean body mass, Metabolic syndrome.

Published online: January 02, 2021; PII: S097475591600268

$\mathrm{O}$ besity is recognized as a common chronic health problem in childhood cancer survivors, and cardiovascular disease has been shown to occur at an earlier age in survivors of childhood cancer [1-4]. As acute lymphoblastic leukemia (ALL) is the commonest childhood cancer, we aimed to study the prevalence of obesity and sarcopenia among survivors of childhood ALL using DEXA scan, as compared to sibling controls, and evaluate association with risk factors.

\section{METHODS}

This study was conducted between July, 2013 and June, 2014 at the pediatric hemato-oncology department of our comprehensive cancer center. The study participants (cases) included survivors of childhood ALL who were less than 18 years of age at diagnosis, treated between 1996 and 2008, in first complete remission, and were at least two years after completion of therapy. Of the total 207 ALL patients that were treated, 122 were eligible for the study and we were able to contact and obtain consent from 65 children and young adult survivors (52 males). The treatment protocol was based on a Berlin Frankfurt
Munster (BFM) backbone that included a four-drug induction and prednisolone as the glucocorticoid [5]. Their results were compared with 50 healthy sibling controls that were matched for age ( \pm 1 year) and sex. The controls were determined to be healthy and eligible for the study if there was no evidence of any medical illness on detailed history and physical examination. The study was approved by the institutional ethics committee and conducted after obtaining informed consent from the study participant or parents (in case of minor).

Details of method of measuring anthropometric indices (weight, height, BMI) and physical activity quotient (PAQ) have been previously published [5]. Three-compartment body composition was assessed using DEXA and Hologic Explorer (S/N 91531) software version 13.3:3. The DEXA machine directly generates absolute values as well as $Z$ scores for bone mineral content, and lean body mass (LBM) measures, as the National Health and Nutrition Examination Survey (NHANES) reference data is integrated into the software.

Study participants' body mass index (BMI) status was determined by using WHO BMI growth charts and 
categorized as normal weight (BMI $>5$ th to $<85$ th percentile), overweight ( $>85$ th to $<95$ th percentile), or obese (BMI $\geq 95$ th percentile) [6,7]. High adiposity was defined as body fat percentage (BF\%) levels higher than the 85th percentile of McCarthy $\mathrm{BF} \%$ reference data (NHANES) for each age-sex group (overweight $\geq 85$ th percentile and obesity $\geq 95$ th percentile) $[8,9]$. Sarcopenia was defined as LBM $<5$ th percentile of reference data (NHANES). Sarcopenic obesity was defined as participants fulfilling criteria for sarcopenia and obesity by BF\% and LBM [8]. Body fat mass index (BFMI) and lean body mass index (LBMI) were calculated from DEXA-measured body-composition data as BF or LBM in $\mathrm{kg}$ per $\mathrm{m}^{2}$ height.

Statistical analyses: The body compositions of two groups such as weight, height, BMI, BF \% etc. were compared using independent $t$ test and chi-square test. Analysis of variance (ANOVA) was used to compare delta BMI change among thin, average and overweight patients at diagnosis. Univariate and multivariate logistic regressions were used to study the influence of various demographic and disease-related factors for obesity and sarcopenia among the survivors. The results were interpreted using odds ratio (OR) and 95\% confidence interval (CI). The Statistical Package for Social Sciences for Window's software (IBM SPSS Statistics version 23.0) was used for all analyses. Significance was set at $P<0.05$.

\section{RESULTS}

At the time of evaluation, the 65 study participants [median (range) age, 15 (7.7-27.5) years] were median (range) 4.3 (2-14.8) years from treatment completion, and had a median (range) follow-up of 7.2 (5-17.2) years.

The values for body composition for the survivors and the control groups are provided in Table I. The mean (SD) $\mathrm{BF} \%$ was significantly higher among ALL survivors as compared to the controls [35.2 (7.4) vs 30.2 (8.0); $P=0.001]$, and a similar trend was observed when analyzed by gender. Using $\mathrm{BF} \%$, obesity was observed among $21.5 \%(14 / 65)$ of study participants and none of the controls $(P=0.00)$ and overweight among 55\% (36/65) and $48 \%(24 / 50)$ of cases and controls respectively $(P=0.7)$. On the other hand BMI under-estimated obesity $(6 \%, 4 /$ $65, P=0.02)$ and overweight $(26 \%, 17 / 65, P=0.01)$ among the study participants. It also detected fewer control participants to be overweight $(6 \%, 3 / 50, P=0.01)$.

We further looked at WHO BMI $z$-scores at diagnosis and noted that $10 / 65(15 \%)$ were underweight/thin, 9/65 (14\%) were overweight, and none was obese. While at evaluation, these proportions changed to $26.1 \%(n=17)$ overweight and $6.1 \%(n=4)$ obese, respectively. No participant was underweight/thin. Delta BMI change was calculated from baseline BMI percentile, which revealed that the highest mean (SD) delta change occurred among thin [2067(882)] followed by normal [273(50)] and overweight [45(30)] patients, $(P<0.001)$.

The absolute values for LBM and LBMI for the study participants' were lower than the controls but this was not statistically significant $(P=0.38,0.68)$ (Table I). The prevalence of sarcopenia was similar in both the groups $(39 / 65,60 \% ; 28 / 50,56 \%)$, respectively $(P=0.10)$. However, the female survivors $(11 / 13,85 \%)$ were more sarcopenic compared to their male counterparts $(28 / 52,54 \%)$ $(P=0.05)$. Sarcopenic obesity was observed among $14 \%$ $(9 / 65)$ of study participants and none of the controls $(P=0.00)$. Of these 9 patients, 1 was thin and none were overweight or obese based on baseline BMI, this difference was not statistically significant $(P=0.29)$.

Distribution of demographic, disease and treatment exposure among participants who were obese or sarcopenic is displayed in Table II. The study participants had a 28 times higher odds of being obese compared to the controls [OR ( $95 \% \mathrm{CI}) 28$ (1.7 to 490); $P=0.002$ ] . On univariate logistic regression analysis female gender, age at diagnosis less than 10 years, $\mathrm{T}$ immunophenotype, high NCI risk, receiving cranial irradiation, more than 5 years since therapy, younger age at evaluation, being sarcopenic, and PAQ $>2$ were significantly associated with obesity. On multi-variate analysis, only female gender [OR $(95 \% \mathrm{CI}) 7.3(1.1-50.1) ; P=0.04]$, high NCI risk category [OR $(95 \% \mathrm{CI}) 6.7(1.1-43.5) ; P=0.04]$, cranial

Table I Body Composition by DEXA Scan and Anthropometry of Survivors of Childhood Acute Lymphoblastic Leukemia and Sibling Controls $(N=115)$

\begin{tabular}{lllr}
\hline Characteristics & $\begin{array}{l}\text { ALL survivors } \\
(n=65)\end{array}$ & $\begin{array}{l}\text { Controls } \\
(n=50)\end{array}$ & Pvalue \\
\hline Weight, $\mathrm{kg}$ & $50.9(16)$ & $48.5(16.3)$ & 0.42 \\
Height, $\mathrm{cm}$ & $154.6(15.3)$ & $156(14.9)$ & 0.61 \\
BMI, $\mathrm{kg} / \mathrm{cm}^{2}$ & $20.8(3.7)$ & $19.3(3.8)$ & 0.03 \\
Body fat, \% & $35.2(7.4)$ & $30.2(8.0)$ & $<0.001$ \\
BFMI & $7.26(2.4)$ & $5.8(2.3)$ & $<0.001$ \\
Lean body mass & $30355.9(9886.1)$ & $32092.7(10644.6)$ & 0.38 \\
LBMI & $12.3(2.1)$ & $12.5(2.5)$ & 0.68 \\
Trunk/Leg fat \% & $0.9(0.6)$ & $0.8(0.5)$ & 1.0 \\
Trunk lean mass & $14861(5000.9)$ & $15835.93(5509.8)$ & 0.33 \\
\hline $\begin{array}{l}\text { All values in } \\
\text { absorptiometry; ALL: Acute lymphoblastic leukemia; BMI: Body }\end{array}$ \\
$\begin{array}{l}\text { mass index at evaluation; BF\%: Body fat percentage; BFMI: Body } \\
\text { fat mass index; LBMI: Lean body mass index. }\end{array}$
\end{tabular}


Table II Demographic and Disease Characteristics of Obese and Sarcopenic Survivors of Childhood Acute Lymphoblastic Leukemia (All)

\begin{tabular}{lllr}
\hline Characteristic & $\begin{array}{c}\text { Survivors } \\
(n=65)\end{array}$ & $\begin{array}{l}\text { Obese } \\
(n=14)\end{array}$ & $\begin{array}{c}\text { Sarcopenic } \\
(n=39)\end{array}$ \\
\hline Male & $52(80)$ & $6(42.9)$ & $29(74.4)$ \\
Age at diagnosis $<10 \mathrm{y}$ & $19(29.2)$ & $14(100)$ & $26(66.7)$ \\
T cell immunophenotype & $14(21.5)$ & $4(28.6)$ & $8(20.5)$ \\
High NCI risk & $30(46.2)$ & $5(35.7)$ & $19(48.7)$ \\
Cranial radiation & $31(47.7)$ & $4(28.6)$ & $19(48.7)$ \\
$>5$ y after therapy & $29(44.6)$ & $4(28.6)$ & $19(48.7)$ \\
Age at evaluation $<10 y$ & $10(15.4)$ & $6(42.9)$ & $7(17.9)$ \\
High BMI & $21(32.3)$ & $14(100)$ & $1(2.6)$ \\
Sarcopenic LBM & $39(60)$ & $9(64.3)$ & $39(100)$ \\
Normal PAQ score & $21(32.3)$ & $13(92.9)$ & $27(69.2)$ \\
\hline
\end{tabular}

All values in no. (\%). DEXA: Dual energy X-ray absorptiometry; NCI: National Cancer Institute; BMI: Body mass index at evaluation; LBM: Lean body mass; PAQ: Physical activity quotient.

irradiation [OR (95\% CI) $9.9(1.2-83.3) ; P=0.04]$, and younger age [OR $(95 \% \mathrm{CI}) 10.2(1.1-91.4) ; P=0.04]$, had a significant association with obesity.

Among 31 children exposed to cranial RT, doses of 12.6 Gy were administered to 27 of whom one was obese. Four children received cranial RT at doses of $18 \mathrm{~Gy}$ and three of them were obese. None of the four obese survivors suffered from endocrinopathies or short stature to account for obesity. None of the baseline characteristics were associated with higher prevalence of sarcopenia.

Table III Multivariate Logistic Regression Analysis of Obese and Sarcopenic Survivors of Childhood Acute Lymphoblastic Leukemia $(N=65)$

\begin{tabular}{llr}
\hline Variables & Odds ratio $(95 \%$ CI) & Pvalue \\
\hline Female & $7.3(1.1-50.1)$ & 0.04 \\
Age at diagnosis $(<10 \mathrm{y})$ & $1.2(0.2-7.5)$ & 0.85 \\
T Immunophenotype & $1.9(0.1-25.6)$ & 0.63 \\
High NCI risk & $6.7(1.1-43.5)$ & 0.04 \\
Cranial radiation & $9.9(1.2-83.3)$ & 0.04 \\
$>5 y$ after therapy & $1.6(0.3-8.6)$ & 0.59 \\
Age at evaluation $(<10 \mathrm{y})$ & $10.2(1.1-91.4)$ & 0.04 \\
High BMI & $2.1(0.4-11.5)$ & 0.38 \\
LBMI-Sarcopenic & $1.3(0.3-5.6)$ & 0.72 \\
PAQ $>2$ & $8.2(0.8-83.3)$ & 0.07 \\
\hline
\end{tabular}

NCI: National Cancer Institute; yr: year; BMI: Body mass index at evaluation; LBM: Lean body mass; LBMI: Lean body mass index; PAQ: Physical activity quotient.

\section{DISCUSSION}

In the present analysis, using DEXA we observed that more than one-fifth of the survivors of childhood ALL were obese and half of them were overweight at a median follow-up of 7 years. A wide variation in prevalence rates of obesity (18-80\%) has been reported among childhood cancer survivors in studies using DEXA scans. The St Jude life time cohort study [10] reported obesity in $63 \%$ male and $85 \%$ female ALL survivors at a mean follow up of 25 years, while Barr, et al. [11] from Canada reported obesity and overweight rates of $12 \%$ and $18 \%$ respectively at a median follow-up of 21 years. The range in findings may be attributed to differences in definitions of obesity, treatment protocol and gluco-corticoid doses, duration of follow-up, ethnicity, and social factors as well as the prevalence of obesity in the normal population of the region. Comparison with a control population helped obviate many of these factors. The main advantage of using sibling controls was to avoid confounding biases due to constitutional and environmental factors.

Published studies form India have used weight- and height-based indices and reported lower prevalence rates of obesity (2.5-12\%) and overweight (19-20\%) [12,13]. In order to be comparable with these data, we adopted the BMI criteria and observed similar rates of obesity $(6 \%)$ and overweight (24\%) among our ALL survivors. Hence, BMI underestimated the prevalence of adiposity as it is unable to identify normal and underweight individuals with high body fat. Blijorg, et al. [14] used total fat percentage as the gold standard, and reported that $42 \%$ of male survivors and $65 \%$ of female survivors were misclassified as non-obese using BMI [9].

It is noteworthy that the prevalence of obesity and overweight was not influenced by the study participants' nutritional status at diagnosis, since the thin and normally nourished children treated for ALL were equally predisposed. However, we observed that the delta change in BMI was highest amongst those who were under-weight/thin at diagnosis, and they probably require close monitoring during follow-up.

Various investigators have described muscle mass loss during the treatment for ALL and its progression throughout therapy and after treatment completion $[15,16]$. This is attributed mainly to the degradation and decreased synthesis of myosin heavy chains, and steroid use, which causes increased glycogen and lipid levels in muscle cells. The prevalence of sarcopenia was equally high amongst our control population, which possibly indicates that ethnically, our population has lower muscle mass compared to Western counterparts. We did; however, observe a high prevalence of sarcopenic obesity 


\section{WHAT THIS STUDY ADDS?}

- Body composition analysis by DEXA scan showed that $21.5 \%$ survivors of childhood ALL were obese and $60 \%$ had sarcopenia on follow-up.

(14\%) among the survivors that was not seen among the controls. This assumes importance in the context of current literature that highlights the combination of the two to be more detrimental and an important contributor to the development of metabolic syndrome [16].

The observed gender difference has previously also been reported $[10,11,17]$. Hyperleptinemia, which occurs in girls during puberty, has been linked to body fat and has been described as a possible mechanism for obesity. Although many investigators have reported the association of cranial RT with obesity, it remains a controversial point [17]. Disturbances influencing the satiety centre and dysfunction of hypothalamic-pituitary axis have been found to cause obesity as well. However, with modern treatment protocols, wherein smaller doses of radiation are delivered with better techniques, recent papers have revealed no association of cranial radiation with the incidence of obesity in survivors of childhood ALL [18]. The increased incidence of obesity among children with NCI high risk disease status may be attributed to the use of higher doses of glucocorticosteroid therapy, poor physical activity and use of cranial radiation in this subset.

The results of our analysis should be interpreted in light of the small sample size and skewed gender ratio, in addition to the fact that influence of unknown psychosocial factors on the controls could not be completely excluded. However, our use of DEXA to accurately identify adiposity and sarcopenia as well as use of matched controls strengthens our findings.

The findings of the present study highlight the high prevalence of obesity and sarcopenic obesity in our population of survivors of childhood ALL. Since these are believed to be forerunners of cardio-metabolic syndrome our results emphasize the need for early recognition and aggressive preventive strategies. Larger interventional studies may identify strategies that have an impact on reducing obesity in this sub-population of children.

Acknowledgements: Dr Uma Athale and Dr Julia Challinor in editing and proof-reading the manuscript.

Ethical clearance: Institutional Review Board; No. RGCIRC/ IRB/77/2013, dated 10 September, 2013.

Contributors: PM: data acquisition, interpretation, drafting the manuscript; GK: study design, data interpretation, reviewing and finalizing manuscript; SJ: data interpretation, reviewing

INDIAN PEDIATRICS manuscript; SJ: data acquisition, reviewing manuscript; AS: data analysis and interpretation, reviewing manuscript.

Funding: None; Competing interests: None stated.

\section{REFERENCES}

1. Tamilselvan St, Scott JX, Sneha L, Divyalakshmi J. Metabolic syndrome in childhood cancer survivors. J Clin Oncol. 2017;35:145.

2. Chueh HW, Yoo JH. Metabolic syndrome induced by anticancer treatment in childhood cancer survivors. Ann Pediatr Endocrinol Metab. 2017;22:82-9.

3. Barnea D, Raghunathan N, Friedman DN, Tonorezos ES. Obesity and metabolic disease after childhood cancer. Oncology (Williston Park). 2015;29:849-55.

4. Commission on ending childhood obesity: Taking action on childhood obesity report. 2018;WHO/NMH/PND/ECHO/ 18.1 .

5. Jain S, Jain S, Kapoor G, Virmani A, Bajpai R. No impact of disease and its treatment on bone mineral density in survivors of childhood acute lymphoblastic leukemia. Pediatr Blood Cancer. 2017;64.

6. World Health Organization. World Health Organization Child Growth Standards. 2006. Accessed March 5, 2012. https://www.who.int/childgrowth/en/

7. Krebs NF, Himes JH, Jacobson D, et al. Assessment of child and adolescent overweight and obesity. Pediatrics. 2007;120:S193-228.

8. McCarthy HD, Cole TJ, Fry T, Jebb SA, Prentice AM. Body fat reference curves for children. Int J Obes (Lond). 2006;30:598-602.

9. Ogden CL, Li Y, Freedman DS, Borrud LG, Flegal KM. Smoothed percentage body fat percentiles for U.S. children and adolescents, 1999-2004. Natl Health Stat Report. 2011;1-7.

10. Karlage RE, Wilson CL, Zhang N, et al. Validity of anthropometric measurements for characterizing obesity among adult survivors of childhood cancer: A report from the St. Jude Lifetime Cohort Study. Cancer. 2015;121: 2036-43.

11. Marriott CJC, Beaumont LF, Farncombe TH, et al. Body composition in long-term survivors of acute lymphoblastic leukemia diagnosed in childhood and adolescence: A focus on sarcopenic obesity. Cancer. 2018;124:1225-31.

12. Prasad M, Arora B, Chinnaswamy G, et al. Nutritional status in survivors of childhood cancer: Experience from Tata Memorial Hospital, Mumbai. Indian J Cancer. 2015;52:219-23.

13. Mohapatra S, Bansal D, Bhalla AK, et al. Is there an increased risk of metabolic syndrome among childhood acute lymphoblastic leukemia survivors? A developing country experience. Pediatr Hematol Oncol. 2016;33:136-49.

Volume 58-MaY 15, 2021 
14. Blijdorp K, van den Heuvel-Eibrink MM, Pieters R, et al. Obesity is underestimated using body mass index and waist-hip ratio in long-term adult survivors of childhood cancer. PLoS One. 2012;7:e43269.

15. Orgel E, Mueske NM, Sposto R, et al. Limitations of body mass index to assess body composition due to sarcopenic obesity during leukemia therapy. Leuk Lymphoma. 2018;59:138-45.

16. Rayar M, Webber CE, Nayiager T, Sala A, Barr RD. Sarcopenia in children with acute lymphoblastic leukemia.
J PediatrHematol Oncol. 2013;35:98-102.

17. Garmey EG, Liu Q, Sklar CA, et al. Longitudinal changes in obesity and body mass index among adult survivors of childhood acute lymphoblastic leukemia: A report from the Childhood Cancer Survivor Study. J Clin Oncol. 2008;26:4639-45.

18. Withycombe JS, Post-White JE, Meza JL, et al. Weight patterns in children with higher risk ALL: A report from the Children's Oncology Group (COG) for CCG 1961. Pediatr Blood Cancer. 2009;53:1249-54.

\section{CLIPPINGS}

Coronavirus infections in the nervous system of children: A scoping review making the case for longterm neurodevelopmental surveillance (Pediatric Neurol. 2021;117:47-63)

This scoping review included 31 studies illustrating nervous system involvement by SARS-CoV-2 virus and 21 studies describing neurological involvement by other human coronaviruses. 31 SARS-CoV-2 articles (27 case reports and 4 case series) portrayed a wide spectrum of neurological manifestations in children involving both central nervous system (ADEM, encephalitis, seizures, stroke) as well the peripheral nervous system (GBS, transverse myelitis). Another group of children presenting with neurological manifestations with COVID-19 were those with MIS-C (multisystem inflammatory syndrome in children) which were described in 8 case reports. The authors found a wide variability in duration of follow-up and extent of evaluation amongst these studies. This study raises a rational concern that these children are at risk of long-term neurodevelopmental deficits which may not be apparent before discharge or during early follow-up. A comprehensive list of signs of potential neuro-developmental impairment across various age groups is also provided by the authors. As underscored by this article, there is a critical need for long-term neuro-developmental follow-up of these children by attaching them to developmental clinics under the collaborative care of pediatricians, pediatric neurologists and child psychologists.

A double-blind randomized, placebo-controlled clinical study of trofinetide in the treatment of fragile $\mathbf{X}$ syndrome (Pediatric Neurol. 2020;110:30-41)

Fragile X syndrome (FXS) is a neuro-developmental disorder characterised by $>200$ CGG repeats in the FMR1 gene. It has a significant prevalence of about 1 in 4000 males and 1 in 6000 females and there is no approved drug therapy for this disorder currently. This randomised controlled trial evaluated safety and tolerability of trofinetide in 72 adolescent and adult males with FXS. Trofinetide is an oral drug which is an analogue of amino- terminal tripeptide of insulin-like growth factor (IGF-1) which is postulated to improve symptoms of FXS by reducing neuroinflammation, normalize dendritic morphology, reduce microglia activation and astrogliosis. Subjects were randomized to receive $35 \mathrm{mg} / \mathrm{kg}$ and $70 \mathrm{mg} / \mathrm{kg}$ trofinetide vs placebo BID for 28 days. Both doses were well tolerated and were found safe. Higher dose trofinetide was found to be efficacious in reducing key symptoms of FXS. As the duration of study was short and sample size was limited, larger trials are required to explore the efficacy of this potentially promising drug.

Clinical and imaging features of children with y autoimmune encephalitis and MOG antibodies (Neurol Neuroimmunol Neuroinflamm. 2020;7:e731)

MOG abs (myelin oligodendrocyte glycoprotein antibodies) have been described typically in childhood central nervous system demyelinating disorders. Recently, these antibodies have been reported to be associated with autoimmune encephalitis (AE) with MRI features such as cortical and deep grey matter involvement in children and adults. This study describes a cohort of 10 children with $\mathrm{AE}$ and MOG abs. They presented at a median age of 8 years (range: 4-16 years) with encephalopathy $(10 / 10)$ and a combination of headache, seizures and focal neurologic signs. Contrary to demyelinating disorders, none of the children had white matter involvement except juxta-cortical signal alterations in 6/10 children while all had cortical and deep grey matter involvement. Eight out of 10 children were treated with high dose intravenous methylprednisolone pulse therapy for 3-5 days and 1 child was given IV immunoglobulins. Nine out 10 children had favourable outcome (modified rankin scale 1) at 4 weeks except one child who had a residual focal deficit and had not received immunomodulation at the time of acute illness. This study highlights the crucial need of testing for MOG abs in all the children presenting with autoimmune encephalitis and prompt treatment with immunomodulation in such cases which is pivotal for a favourable outcome.

JUHI GUPTA juhiguptadr@gmail.com 